\title{
Vectorial structure of the near-wall premixed flame
}

\author{
Peipei Zhao and Lipo Wang* \\ UM-SJTU Joint Institute, Shanghai Jiaotong University, Shanghai 200240, China
}

\author{
Nilanjan Chakraborty \\ School of Engineering, Newcastle University, Newcastle-Upon-Tyne, NE1 7RU, United Kingdom
}

(Received 6 November 2018; published 12 June 2019)

\begin{abstract}
The turbulent premixed flame-wall interaction (FWI) has been numerically analyzed in a head-on quenching configuration at statistically stationary state under different boundary conditions. When the wall temperature is not high enough, the near wall flame isosurfaces become broken because of local flame extinction within the quenching and influence zones of FWI. According to the relative orientation of the flame normal and the wall normal, the flame can be either head-on or entrained. Outside of the influence zone the head-on flame elements are predominant, while inside the quenching zone flames are more likely to be entrained. The alignment relations among important vectors, including the principal axes of the strain rate tensor, the flame normal vector and vorticity have been investigated. Outside of the influence zone the normal vector of the progress variable isosurfaces aligns with the most extensive principle axis in the reaction zone and with the most compressive axis in the unburned and burned gas regions. Within the quenching zone, the preferential orientation with the most extensive principal axis disappears, once the flame dilatation and flame-normal strain rate decrease. Conditional alignment statistics demonstrate the distinctly different properties between the entrained flame and the head-on flame parts. In summary, the alignment relations are primarily determined by the relative strengths of chemical heat release, turbulent staining, and wall heat flux.
\end{abstract}

DOI: 10.1103/PhysRevFluids.4.063203

\section{INTRODUCTION}

The wall boundary condition is of essential importance in combustion science and technology, especially from the consideration of down-sizing the combustors. Meanwhile, because of the interference of wall boundary conditions, the combustion processes become more complex than the boundary free cases. In confined space flame-wall interaction (FWI) can lead to strong influences on the combustion performance and device lifetime. Although the boundary free flames have been extensively studied in the existing literature [1-4], the wall effects on the turbulent flames are not sufficiently addressed yet. For instance, in the near wall region because of weakened flame wrinkling and the local flame quenching by the wall heat loss, the interaction between flame and turbulence can lead to new and complex physics. Therefore, it is important to understand FWI in both fundamental and application aspects, such as combustor design and optimization, pollutant emission control and model development.

Poinsot et al. [5] studied the turbulent premixed flame interaction with an isothermal wall based on two-dimensional direct numerical simulations (DNS). Correlations between the flame-wall distance and flame properties indicated that the wall acts as a strong sink for the flame surface density. Gruber et al. [6] studied a $V$-shaped premixed flame anchored in fully developed channel

*lipo.wang@sjtu.edu.cn 


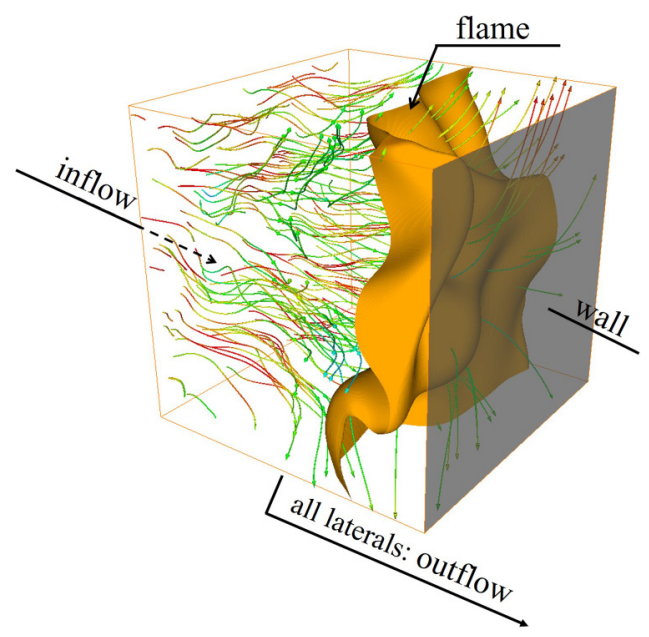

FIG. 1. The statistically stationary FWI configuration. The streamlines are colored with the velocity magnitude.

flow. It shows that the flame thickness changes with the wall-normal distance. Specifically, in the channel center's core region the flames are typically thin flamelets, while near the wall the flames are thickened. To develop the wall flamelet model in turbulent combustion, Bruneaux et al. [7] investigated the evolving turbulent premixed flame propagating towards the wall. From the flame surface density equation, the budget of different terms has been analyzed at different stages of the evolution process. The results suggest that large gradients of flame surface density near the wall are responsible for the dominant transport terms. Lai and Chakraborty [8] analyzed the statistical behavior of the scalar dissipation rate (SDR) of reaction progress variable $c$ to modify the SDR-based closure for the mean reaction rate in the context of Reynolds averaged Navier-Stokes simulations. It is found that the terms of the SDR transport equation at the quenching stage near the wall shows distinctly different contribution from that away from the wall. Recently Zhao et al. $[9,10]$ introduced a statistically stationary FWI configuration as shown in Fig. 1. The fresh reactant stream is continuously fed from the inflow side and flows out from the lateral boundaries. The spatial turbulent flame is anchored by the solid wall boundary to conform with the practical working conditions. Geometrically the distorted flames generally consist of the head-on flame part and the entrained flame part. The DNS results of the dependence of flame temperature and wall heat flux on the flame wall distance can be reasonably quantified by a simplified counterflow model [9].

The transport equations of the scalar gradient $c_{i}=\frac{\partial c}{\partial x_{i}}[11]$ and SDR defined as $\chi=2 D\left(c_{i} \cdot c_{i}\right)$ [12-16] can be respectively written as

$$
\frac{D c_{i}}{D t}=-c_{j} \frac{\partial u_{j}}{\partial x_{i}}+\frac{\partial}{\partial x_{i}}\left\{\frac{1}{\rho}\left[\dot{w}+\frac{\partial}{\partial x_{k}}\left(\rho D \frac{\partial c}{\partial x_{k}}\right)\right]\right\}
$$

and

$$
\frac{D \chi}{D t}=-4 D c_{i} c_{j} s_{i j}+4 D c_{i} \frac{\partial}{\partial x_{i}}\left\{\frac{1}{\rho}\left[\dot{w}+\frac{\partial}{\partial x_{k}}\left(\rho D \frac{\partial c}{\partial x_{k}}\right)\right]\right\}
$$

where $s_{i j}=\frac{1}{2}\left(\frac{\partial u_{i}}{\partial x_{j}}+\frac{\partial u_{j}}{\partial x_{i}}\right)$ is the strain rate tensor. The term $-4 D c_{i} c_{j} s_{i j}$ in Eq. (2) represents the action of flame normal straining on the scalar dissipation rate transport. It can be shown that

$$
\frac{c_{i} c_{j} s_{i j}}{|\nabla c|^{2}}=s_{n}=\Lambda_{i} \cos ^{2}\left\langle\vec{n}, \vec{\lambda}_{i}\right\rangle=s_{n_{-} 1}+s_{n_{-} 2}+s_{n-3},
$$


where $\Lambda_{i}(i=1,2,3)$ are the principal strain rates, $s_{n_{-} 1}, s_{n_{-} 2}$, and $s_{n \_3}$ represent the contributions to flame-normal strain rate along principle axes $\vec{\lambda}_{i}$ of the strain rate tensor.

In addition to the SDR, the dissipation rate of turbulent kinetic energy is closely related to the enstrophy $\Omega=\omega_{i} \omega_{i} / 2$ (where $\omega_{i}$ is the $i$ th component of the vorticity vector $\vec{\omega}$ ) [17-20] and thus the closure of dissipation rate of turbulent kinetic energy in FWI depends significantly on the fundamental understanding of the enstrophy. The transport equation of the enstrophy for premixed turbulent flames is given by [17-20]

$$
\frac{D \Omega}{D t}=\omega_{i} s_{i k} \omega_{k}+\frac{\epsilon_{i j k} \omega_{i}}{\rho^{2}} \frac{\partial \rho}{\partial x_{j}}\left(\frac{\partial p}{\partial x_{k}}-\frac{\partial \tau_{k l}}{\partial x_{l}}\right)+\frac{\epsilon_{i j k} \omega_{i}}{\rho} \frac{\partial^{2} \tau_{k l}}{\partial x_{j} \partial x_{l}}-2 \Omega \frac{\partial u_{k}}{\partial x_{k}},
$$

where $\tau_{k l}=\mu\left(\frac{\partial u_{k}}{\partial x_{l}}+\frac{\partial u_{l}}{\partial x_{k}}\right)-2 / 3 \mu \delta_{k l} \frac{\partial u_{m}}{\partial x_{m}}$ is the component of the viscous stress tensor. The first term on right-hand side is the vortex stretching contribution to the enstrophy transport, which can be expressed as

$$
\omega_{i} s_{i k} \omega_{k}=2 \Omega \Lambda_{i} \cos ^{2}\left\langle\vec{\omega}, \vec{\lambda}_{i}\right\rangle
$$

It can be seen from Eq. (5) that the vortex-stretching term behavior of the enstrophy transport depends on the relative alignment of the vorticity vector with local principal strain rates.

It should be evident from Eqs. (3) and (5) that the alignments of $\nabla c$ and $\vec{\omega}$ with local principal strain rate eigendirections (i.e., principal axis) determine the statistical behaviours flame normal strain rate contribution (alternatively scalar-turbulence interaction) $-4 D c_{i} c_{j} s_{i j}$ in the SDR transport equation (2) [12] and the vortex-stretching term $\omega_{i} S_{i k} \omega_{k}$ in the enstrophy transport equation (4) [17-20], respectively. These involved vectors in the above equations, including the scalar gradient, principal axes of the strain rate tensor and vorticity, are important to the fundamental understanding and modeling of dissipations of scalar and velocity fluctuations. As the relative alignments of the scalar gradient, principal axes of the strain rate tensor and vorticity is the main focus of this analysis, the transport of the SDR and enstrophy and the other terms in their transports will not be investigated further in this paper.

The vector alignment relations for the boundary free case have been extensively studied in both nonreacting flows [21-24] and reacting flows [13,15,25-33]. Fluid dynamics in boundaryfree reactive turbulence depends on the relative contributions from the nonlinearly coupled heat release and turbulent motion [16,32]. It has been identified that in nonreacting flows the passive scalar gradient preferentially aligns with the most compressive strain axis, which explains also the production of scalar gradients. However, in reactive flows the flame normal prefers to align with the most extensive strain axis when the strain rate due to flame normal acceleration arising from heat release overcomes turbulent straining [12,15,16,32,33]. More detailed results under different thermal and turbulent conditions, including turbulent intensity [32], Lewis number [16], and Damköhler number [13], can also be found in the existing literature.

A number of previous analyses [25-27] analyzed the alignment statistics of the vorticity vector with local principal strain rates in nonpremixed flames and demonstrated that the vorticity vector $\vec{\omega}$ aligns with the intermediate principal strain rate in nonpremixed flames similar to the nonreacting turbulent flows [21,32,34-40]. However, an appreciable alignment between the vorticity vector and the most extensive principal strain rate has been observed in the places of high heat release [25-27], whereas $\vec{\omega}$ remains mostly perpendicular to the most compressive principal strain rate in both reactive and nonreactive regions of nonpremixed turbulent combustion. Hamlington et al. [32] analyzed the alignment of vorticity with local principal strain rates in premixed flames in the thin reaction zones regime combustion, and reported that the alignment of $\vec{\omega}$ with local principal strain rates in the thin reaction zones flames is qualitatively similar to the previous findings in the context of nonpremixed combustion [25-27] (i.e., principal alignment with the intermediate principal strain rate and an increased alignment with the most extensive principal strain rate in the heat releasing zone). Recently Chakraborty [19] demonstrated that both global Lewis number Le and the regime of combustion can significantly affect statistical behavior of vorticity alignment with 
local principal strain rates. Furthermore, the relative alignments of $\vec{\omega}$ with the most compressive and extensive principal strain rates depend on the values of Damköhler number and global Lewis number. All the aforementioned analyses for reacting flows were carried out for flows away from wall. Although these aspects for the near-wall case were touched upon in previous studies by Lai and Chakraborty [41] and Lai et al. [20] for an unsteady head-on quenching, the alignment statistics in a statistically stationary flame-wall interaction configuration has never been done. Moreover, the alignment statistics of the reactive scalar gradient and the vorticity vector for the entrained and head-on flame elements have been compared for the first time in this analysis. This physical understanding is pivotal for the model developments for dissipation rates of scalar variance and turbulent kinetic energy in the near-wall region in the case of premixed turbulent flames during flame-wall interaction.

In the rest of the paper, first, numerical implementation details and relevant formulations are presented. Then results related to the flame dynamics and statistics, are discussed. Finally, the main findings are summarized to draw conclusions.

\section{NUMERICAL IMPLEMENTATION AND MATHEMATICAL BACKGROUND}

The compressible three-dimensional Navier-Stokes equations are numerically solved. Here the species field is characterized by a reaction progress variable $c$ defined in terms of reactant mass fraction $Y_{R}$, as $c=\frac{Y_{R, u}-Y_{R}}{Y_{R, u}-Y_{R, b}}$, where $Y_{R, u}$ is the reactant mass fraction in fresh stream and $Y_{R, b}$ represents the reactant mass fraction in the burned gas. According to this definition, $c$ increases from zero in the fresh reactant side to unity in the burned product side. The chemical reaction is represented by an one-step irreversible reaction (Reactants $\rightarrow$ Products) following several previous studies [5,7,20,42-45], which satisfactorily predicted measured experiment values [46,47] of the wall heat flux and flame quenching distance, especially at the low wall temperature [48]. It should be indicated that the quenching of premixed flames near isothermal wall is mainly a result of heat transfer, which can be satisfactorily captured by simple chemistry. Kurdyumov et al. [44] used the one-step irreversible model for the chemical reaction to study the flashback or propagation of premixed flames at the base of a laminar boundary layer of a reactive mixture. They concluded that the critical front velocity, determining the onset of flashback, depends on the wall temperature and flow parameters, such as the Lewis number and Karlovitz number. Recently Lai et al. [49] investigated the statistics of wall heat flux, flame-quenching distance and the flame surface density and scalar dissipation rate based mean reaction rate closures for head-on quenching of statistically planar turbulent premixed flames for both simple and detailed chemical mechanisms. A good agreement has been found for the full detailed chemistry temperature-dependent simulations and the simple chemistry, constant thermo-physical property based simulations. Therefore, the DNS results with simple chemistry can be considered reliable, at least in a qualitative sense in the context of detailed chemistry based analysis. With the nondimensional temperature defined by $\tilde{T}=\frac{T-T_{u}}{T_{a d}-T_{u}}$ ( $T_{a d}$ is the adiabatic flame temperature, $T_{u}$ is the unburned gas temperature), the simplified Arrehenius law governing the chemical kinetics is expressed as $\dot{w}=B \rho(1-c) \exp \left[-\frac{\beta(1-\tilde{T})}{1-\alpha(1-\tilde{T})}\right]$, where $B$ is the pre-exponential factor, $\beta$ is the Zel'dovich number given by $\beta=\frac{T_{a}\left(T_{a d}-T_{u}\right)}{T_{a d}^{2}}\left(T_{a}^{1-\alpha(1-T)}\right.$ is the active temperature), $\alpha$ is the heat release parameters defined with $\alpha=\frac{T_{a d}-T_{u}}{T_{a d}}$.

The initial field is specified using the one-dimensional steady planar flame solutions (see [50]). The computational domain is taken to be a cube with each dimension of $L=70 \delta_{z}$, where $\delta_{z}=D_{t h} / S_{L}$ refers to the Zel'dovich flame thickness with $D_{t h}$ and $S_{L}$ denoting the thermal diffusivity of unburned gas and unstretched laminar flame speed, respectively. On the inflow surface, the velocity is specified as the combination of the mean part $U_{i}$ and the fluctuating part $u_{i}^{\prime}$ by scanning an auxiliary homogeneous isotropic turbulent field generated a priori based on a prescribed energy spectrum [51], by which the turbulence in the domain can be maintained. A uniform $256 \times 256 \times 256$ Cartesian grid can resolve the smallest turbulent scale and ensure about seven grid points per thermal flame thickness $\delta_{t h}=\frac{T_{b}-T_{u}}{|\nabla T|_{\max }}$. The characteristic flow and flame parameters 
TABLE I. Characteristic flow and flame parameters. ${ }^{a}$

\begin{tabular}{lccccccc}
\hline \hline $\operatorname{Pr}$ & $\mathrm{Sc}$ & $\mathrm{Ma}$ & $\mathrm{Ka}$ & $\mathrm{Da}$ & $\gamma$ & $\alpha$ & $\beta$ \\
\hline 0.7 & 0.7 & 0.014 & 2.37 & 1.89 & 1.4 & $2.3 / 3.3$ & 6.0 \\
\hline \hline
\end{tabular}

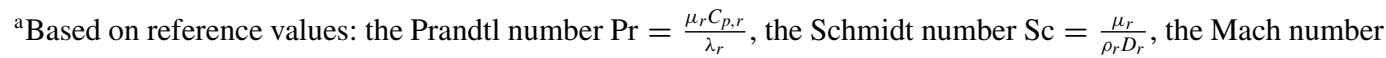
$\mathrm{Ma}=\frac{u_{r}}{a_{r}}\left(a_{r}=\sqrt{\gamma R_{g} T_{u}}\right)$, the Karlovitz number $\mathrm{Ka}=\left(\frac{l_{t}}{\delta_{t h}}\right)^{-1 / 2}\left(\frac{u^{\prime}}{S_{L}}\right)^{3 / 2}$ with $l_{t}$ representing the inflow integral length, the Damköhler number $\mathrm{Da}=\frac{l_{t} / u^{\prime}}{\delta_{t h} / S_{L}}$, and $\gamma$ is the ratio of specific heats. Here the quantities with subscript $r$ denote the reference values.

are listed in Table I. The reference length $l_{r}$ and reference velocity $u_{r}$ are chosen as the domain size $L$ and the laminar flame speed $S_{L}$, respectively; the reference density $\rho_{r}$ and the reference values of other fluid property parameters such as specific heat $C_{p, r}$, thermal conductivity $\lambda_{r}$, and mass diffusivity $D_{r}$ are taken to be the corresponding values of the fresh gas.

The numerical solver is developed based on a parallel three-dimensional DNS code named SENGA [52]. The spatial derivatives are computed using the 10th-order central difference scheme for the internal points, while the scheme order decreases gradually to one-sided second-order at the boundary points. The temporal integration adopts an explicit third-order low-storage Runge-Kutta scheme [53]. The boundary condition setting for the outflow lateral faces follows the three-dimensional Navier-Stokes characteristic boundary condition formulation [54-56]. For comparison, totally we perform five simulations with different inflow mean velocities, including $U_{1} / S_{L}=6.0,8.0$, and 10.0, and different wall boundary conditions, including the adiabatic wall case, intermediate wall temperature case with $\tilde{T}_{w}=0.5$ and cold wall case with $\tilde{T}_{w}=0.0$, which are listed in Table II. For the present counterflow like FWI case, both the turbulence intensity and the length scale change as the flame approaches the wall. Following the boundary layer case, it is possible to define $\operatorname{Re}=U_{1} \delta / \nu$ based on the mean inlet velocity and the so-called boundary layer thickness $\delta \sim \sqrt{v / a}$, where $v$ is the kinematic viscosity and $a=U_{1} / L$ is the strain rate of the mean flow. In order to ensure a converged statistics, the simulation the data has been collected over a duration of three throughpass times (i.e., $3 L / U_{1}$ ) once the simulation reached statistically stationary state. Interested readers are referred to Ref. [9] regarding the statistically stationary state under which the statistics have been extracted.

\section{OVERALL FLAME AND FLOW STRUCTURE}

In FWI, the wall interference has strong influences on the near-wall turbulence intensity, flow temperature, and the flame wrinkling. Figure 2 shows the flame front and the vortex tubes represented by the large positive second invariant of the velocity gradient $\Omega$, which is defined as

$$
\Omega=\frac{1}{2}\left\{\left[\frac{\partial u_{i}}{\partial x_{i}}\right]^{2}-\left(s_{i j} s_{i j}-r_{i j} r_{i j}\right)\right\} .
$$

TABLE II. The cases simulated.

\begin{tabular}{lccr}
\hline \hline Case & Wall boundary condition & Inflow mean velocity & Re \\
\hline 6A & Adiabatic wall & $U_{1} / S_{L}=6.0$ & 23.0 \\
8A & Adiabatic wall & $U_{1} / S_{L}=8.0$ & 30.0 \\
10A & Adiabatic wall & $U_{1} / S_{L}=10.0$ & 33.0 \\
8T5 & $U_{1} / S_{L}=8.0$ & 30.0 \\
8T0 & Isothermal wall with $\tilde{T}_{w}=0.5$ & $U_{1} / S_{L}=8.0$ & 30.0 \\
\hline \hline
\end{tabular}




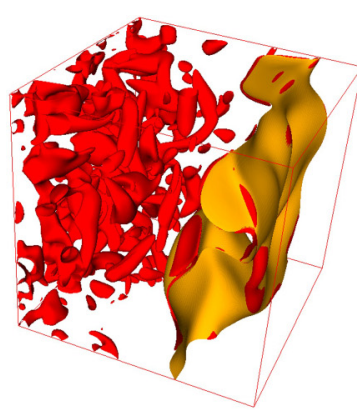

(a)

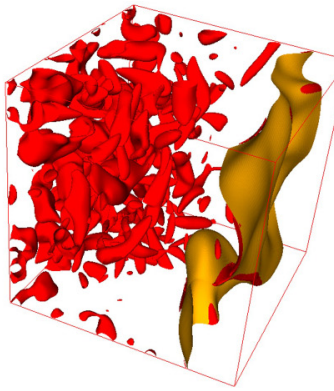

(b)

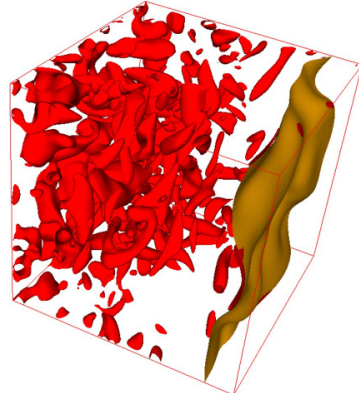

(c)

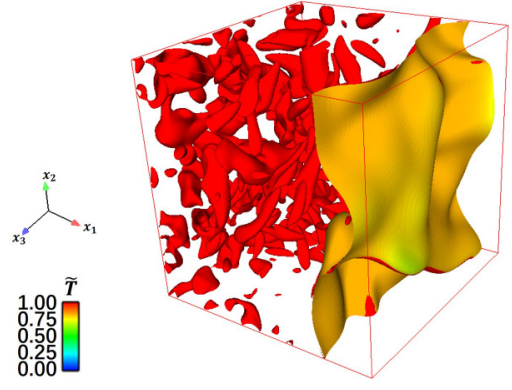

(d)

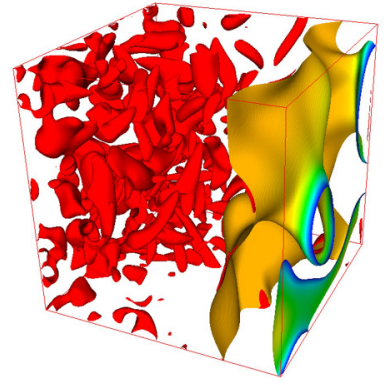

(e)

FIG. 2. Comparison of the flow and flame structure: (a) case 6A, (b) case 8A, (c) case 10A, (d) case 8T5, and (e) case 8T0. The flame is presented by the $c=0.85$ isosurface colored by the local temperature. When the wall temperature decreases, the near-wall flame speed decreases and thus the flame front tends to be closer to the wall. Especially for the $8 \mathrm{~T} 0$ case, the flame front breaks because of local quenching.

Here $r_{i j}=\frac{1}{2}\left(\frac{\partial u_{i}}{\partial x_{j}}-\frac{\partial u_{j}}{\partial x_{i}}\right)$ is the rotational rate tensor element. Since the dilatation rate appears explicitly, Eq. (6) includes density variation (i.e., compressibility) effects and is able to function as a measure where the fluid flow is vorticity dominated $(\Omega>0)$ and where it is strain rate dominated $(\Omega<0)$ for both the incompressible and compressible cases.

In Fig. 2 the flame isosurface, defined as the $c=0.85$ isosurface corresponding to the maximum chemical reaction rate, is colored by the local nondimensional temperature. Small-scale turbulence is largely suppressed between the flame and the wall. For the three adiabatic wall cases in Figs. 2(a)2(c), because of the same spatial distribution of the $c$ and $\tilde{T}$ field variables under the same boundary conditions, the nondimensional flame temperature remains constant as 0.85 . However, with the increase of the mean inflow velocity, the flame is pushed closer to the wall, and flame wrinkling weakens. When the wall temperature decreases, the near-wall flame speed decreases and thus the flame front tends to be closer to the wall, as shown in Figs. 2(b), 2(d), and 2(e). Especially for the $8 \mathrm{~T} 0$ case, the flame front breaks because of local quenching. Defining the flame-wall distance $\delta_{c=0.85}$ as the one from a flame surface point to the wall along the wall normal direction. Quantitatively, the probability density function (PDF) of the normalized flame-wall distance $\delta_{c=0.85} / \delta_{z}$ is shown in Fig. 3. With the increase of the mean inflow velocity, i.e., from case 6Aand 8A to 10A, Fig. 3(a) indicates that the most probable flame-wall distance decreases. Similarly Fig. 3(b) shows when the wall temperature decreases from case 8A and 8T5 to 8T0, the PDF peaks at smaller flame-wall distance, especially for the $8 \mathrm{~T} 0$ case.

On the flame isosurface $c=0.85$, define the unit flame normal vector as $\vec{n}=-\frac{\nabla c}{|\nabla c|}$, pointing from the burned side toward the unburned side. Following the work by Zhao et al. [9], geometrically 


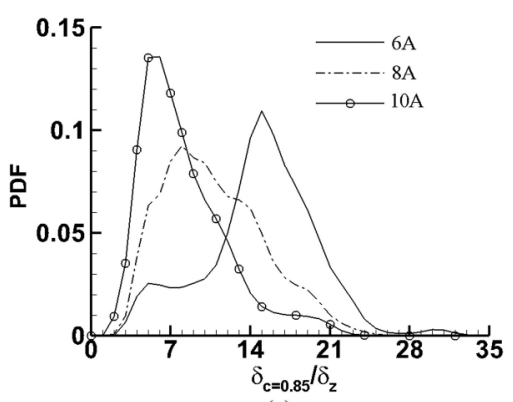

(a)

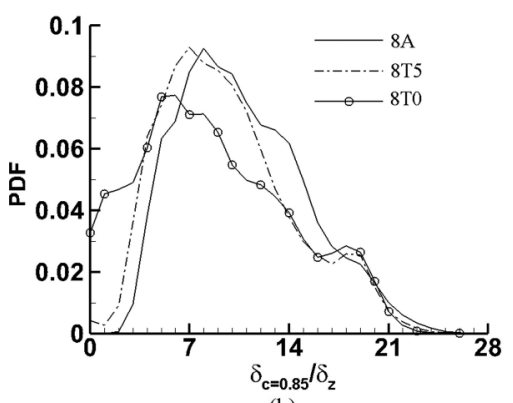

(b)

FIG. 3. PDFs of the flame-wall distance along the wall normal direction for different cases, in accordance with the field visualization in Fig. 2.

a near wall flame front consists of two parts, the head-on flame part with $\vec{n} \cdot \vec{N}>0$ and the entrained flame part $\vec{n} \cdot \vec{N}<0$ with $\vec{N}$ being the unit wall normal vector. As discussed by Poinsot et al. [5] and Zhao et al. $[9,10]$, the vortex pair, or if the local flow moves away or towards the wall, determines the evolution and wrinkling of the flame front. Figure 4 shows the joint PDF between the flame-wall distance and the velocity component $u_{1}$ at the left side of flame brush edge for the case 8T0. For the entrained flame part, the negative velocity component $u_{1}$ is dominant, which means that the entrained flame part is mostly influenced by the vortex-induced back flow, while for the head-on flame brush $u_{1}$ is mostly positive, especially in the quenching zone when the flame-wall distance $\delta_{c=0.8} / \delta_{z}$ is small, which means that flame typically is convected by the flow moving towards the wall.

For the adiabatic wall case, the flame remains unbroken with uniform temperature on the $c$ isosurface. Differently, for the isothermal wall cases with broken flames, the fresh reactant leaks toward the wall boundary, making the head-on and entrained flames much different. Figures 5(a)5 (c) show for case $8 \mathrm{~T} 0$ the joint PDF between the normalized flame temperature $\tilde{T}_{c=0.85}$ and the flame wall distance $\delta_{c=0.85} / \delta_{z}$, conditioned on the overall flame surface, the entrained flame part and the head-on flame part, respectively. According to Poinsot et al. [5], the FWI zone includes two subzones. Specifically, the "quenching zone" is defined as the part from the cold wall to the quenching point, i.e., $\delta_{c=0.85} / \delta_{z} \simeq 2.7$, in which, the flame temperature decreases almost linearly from 0.6 to 0.0 because of the wall heat loss; the "influence zone" is the part in which the flame is influenced by the cold wall boundary and the flame temperature starts to decrease slightly from $\delta_{c=0.85} / \delta_{z} \simeq 8$.0, as shown in Fig. 5(a). Figure 5(b) indicates that the entrained flame part mainly

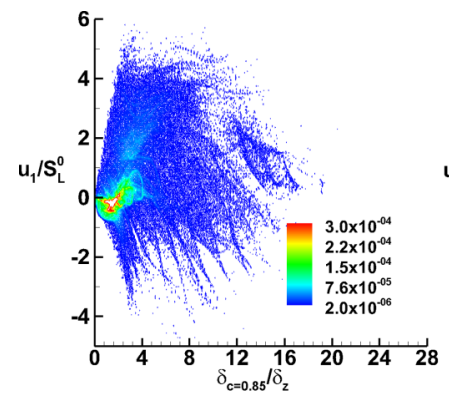

(a)

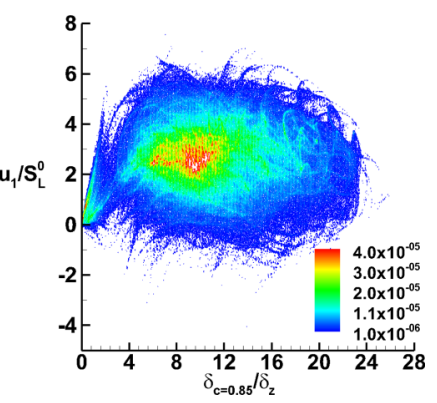

(b)

FIG. 4. Joint PDF between the flame-wall distance and the velocity component $u_{1}$ before the flame brush for (a) the entrained flame elements and (b) the head-on flame elements of case 8T0. 


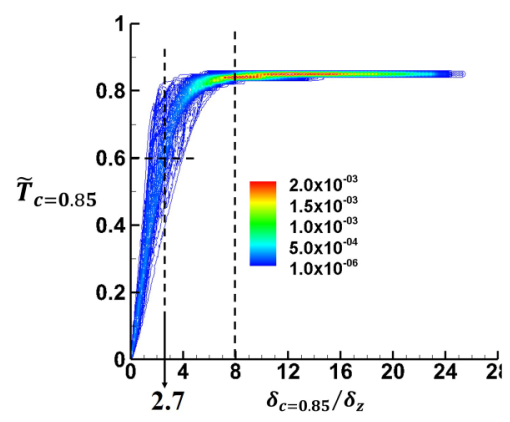

(a)

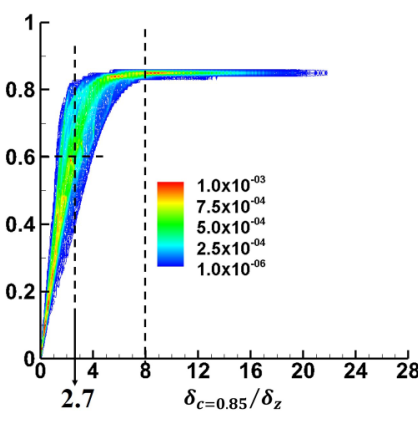

(b)

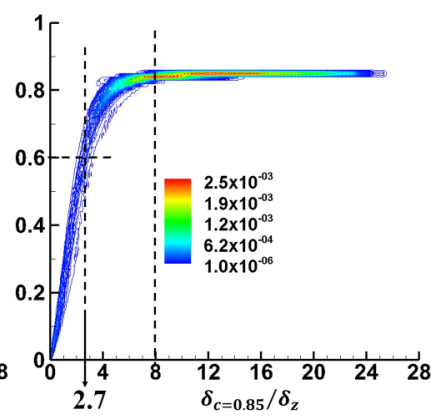

(c)

FIG. 5. Variation of the temperature (on $c=0.85$ ) with the flame-wall distance for (a) the overall sample, (b) the entrained part, and (c) the head-on part of case 8T0.

exists within the influence and quenching zones, while beyond these FWI zones, the head on flame part dominates, as shown in Fig. 5(c). More detailed discussion can be found in Refs. [9,10].

\section{CASE STUDIES}

\section{A. Alignment relations between flame normal and the velocity derivative}

Let $\Lambda_{1}>\Lambda_{2}>\Lambda_{3}$ represent the most extensive, the intermediate and the most compressive strain rates associating with the corresponding principle axes $\vec{\lambda}_{i}$. The PDFs of $\left|\cos \left\langle\vec{\lambda}_{i}, \vec{n}\right\rangle\right|$ for all cases are shown in Fig. 6. This alignment is determined by the competition between the thermal expansion effect and turbulence disturbance. For case 6A, in the reactive regions, e.g., $0.1<c<0.9$ with high heat release rate, the most extensive principle axis $\vec{\lambda}_{1}$ aligns well with $\vec{n}$. From case $8 \mathrm{~A}$ to $10 \mathrm{~A}$ when the incoming flow velocity increases, the alignment between $\vec{\lambda}_{1}$ and $\vec{n}$ becomes weaker
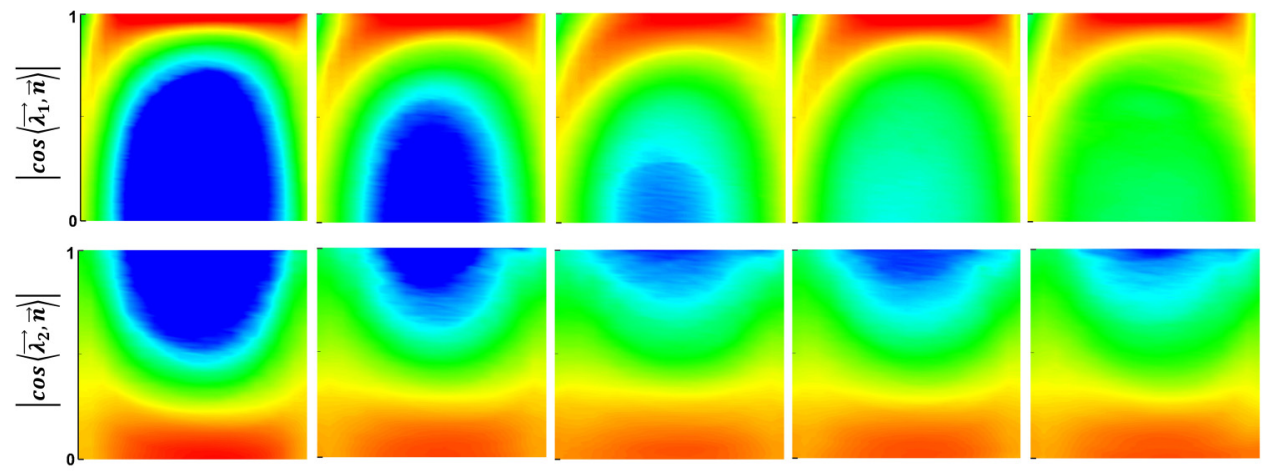

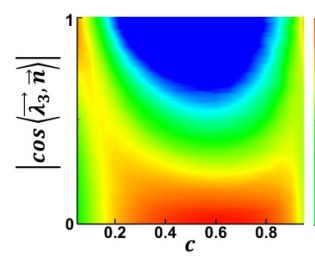

(a)

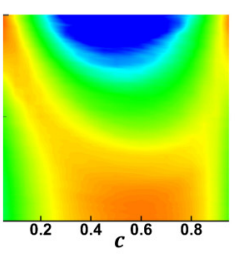

(b)

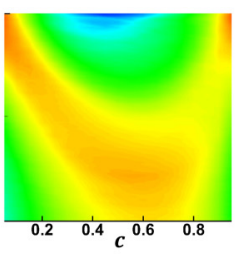

(c)

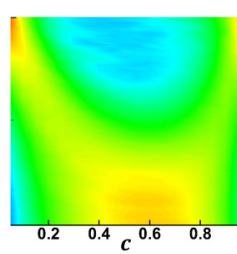

(d)

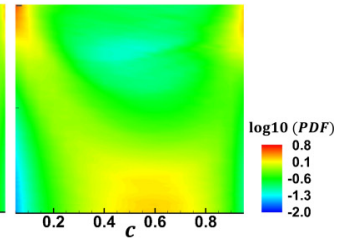

(e)

FIG. 6. PDFs in logarithmic scale of $\left|\cos \left\langle\vec{\lambda}_{i}, \vec{n}\right\rangle\right|$ from $c=0.05$ to $c=0.95$ for (a) case $6 \mathrm{~A}$, (b) case $8 \mathrm{~A}$, (c) case $10 \mathrm{~A},(\mathrm{~d})$ case $8 \mathrm{~T} 5$, and (e) case $8 \mathrm{~T} 0$. 


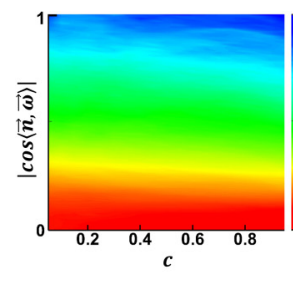

(a)

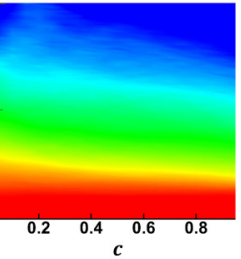

(b)

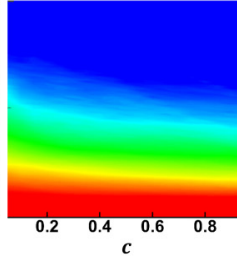

(c)

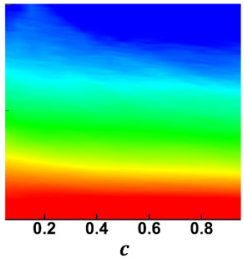

(d)

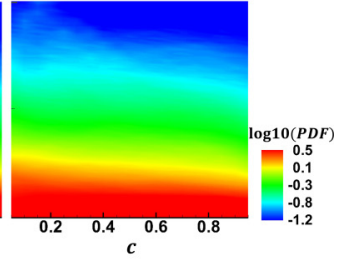

(e)

FIG. 7. PDFs of $|\cos \langle\vec{n}, \vec{\omega}\rangle|$ throughout the flame brush from $c=0.05$ to $c=0.95$ for (a) case 6A, (b) case $8 \mathrm{~A},(\mathrm{c})$ case $10 \mathrm{~A},(\mathrm{~d})$ case $8 \mathrm{~T} 5$, and (e) case $8 \mathrm{~T} 0$.

because of stronger influence from turbulence. From case $8 \mathrm{~T} 5$ to $8 \mathrm{~T} 0$, higher wall heat loss weakens the heat release effect, and thus the alignment between $\vec{\lambda}_{1}$ and $\vec{n}$ weakens as well. In the nonreactive regions with negligible thermal expansion effect, i.e., $c<0.1$ or $c>0.9, \vec{n}$ tends to align with the most compressive principle axis $\vec{\lambda}_{3}$ for all cases, which is similar to the scalar gradient alignment in passive nonreactive scalar turbulence [21,23].

It has been found that $\vec{\omega}$ aligns preferentially with the intermediate principal axis $\vec{\lambda}_{2}$ for both nonreacting flows and premixed flames, with and without the presence of wall [21,32,34-40]. Because $\vec{n}$ aligns with the most extensive axis $\vec{\lambda}_{1}$ and the most compressive axis $\vec{\lambda}_{3}$ in the reactive and nonreactive region, respectively, $\vec{n}$ misaligns with $\vec{\omega}$ because of mutual orthogonality of the eigenvectors, as shown in Fig. 7. From case 6A to 10A, stronger turbulence leads stronger misalignment. Moreover, the wall heat loss does not significantly influence $|\cos \langle\vec{n}, \vec{\omega}\rangle|$, as demonstrated in Fig. 7(b) for case 8A, Fig. 7(d) for 8T5, and Fig. 7(e) for 8T0.

\section{B. Flame strain rates}

In reactive flows heat release induces flame dilation $\Delta=\frac{\partial u_{i}}{\partial x_{i}}$, and influences strongly the flamenormal strain rate $s_{n}=n_{i} n_{j} s_{i j}$ and flame-tangential strain rate $s_{t}=\left(\delta_{i j}-n_{i} n_{j}\right) s_{i j}$. Figure 8 shows the conditional means of these three quantities on different $c$ isosurfaces varying from $c=0.05$ to $c=0.95$ for different cases. For the adiabatic wall cases the dilatation $\Delta$ profiles are almost identical because of the unchanged local heat release rate. For the $8 \mathrm{~T} 5$ and $8 \mathrm{~T} 0$ cases with wall heat loss, the profiles have similar shape but the magnitude drops as the wall heat loss increases.

Qualitatively $s_{n}$ changes with $c$ in a similar manner, i.e., $s_{n}$ reaches the maximum around $c=$ 0.65 , while in the fresh reactant $c \sim 0$ and burned product $c \sim 1$ regions $s_{n}$ is smaller. For the nonadiabatic wall cases locally negative $s_{n}$ appears.

The conditional mean flame-tangential strain rate $s_{t}$ increases from the fresh reactant side to the product side. In the present counterflow like configuration, the higher mean velocity (from adiabatic
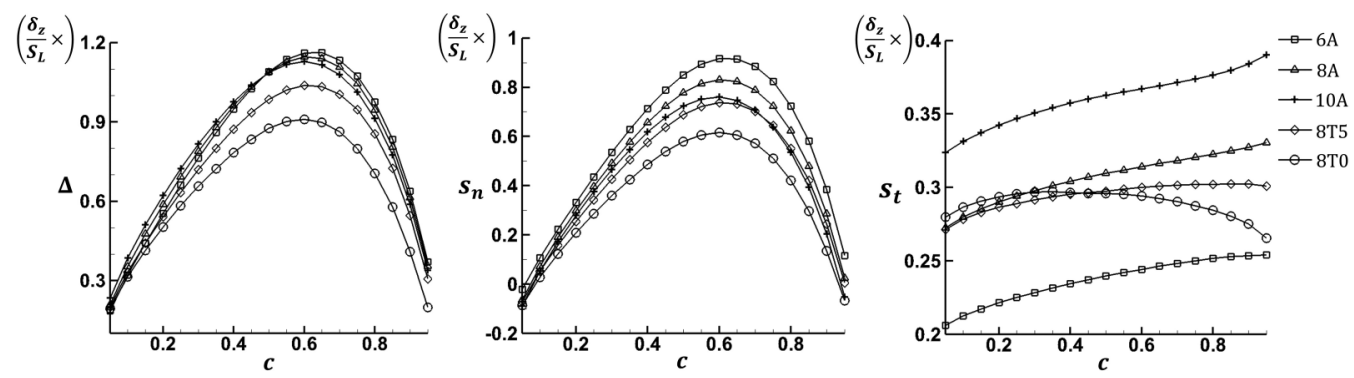

FIG. 8. Conditional mean dilatation $\Delta$, flame-normal strain rate $s_{n}$ and flame-tangential strain rate $s_{t}$ on different $c$ isosurfaces from $c=0.05$ to $c=0.95$ for all cases. 


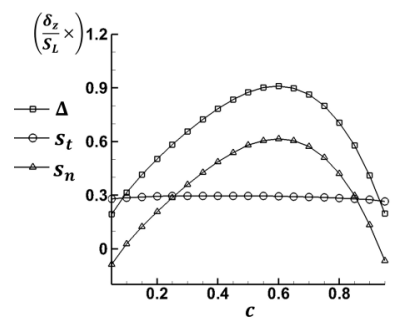

(a)

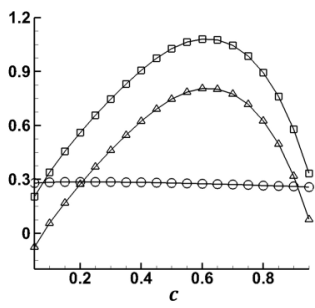

(b)

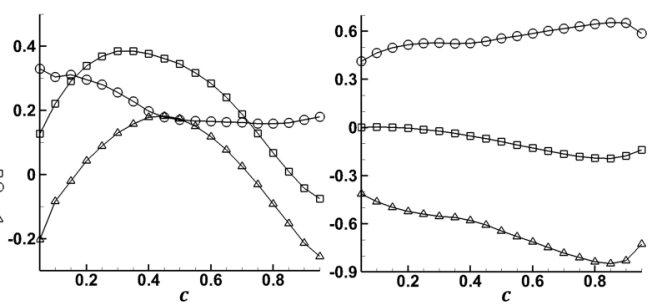

(c)

(d)

FIG. 9. For the cold wall case $8 T 0$ in the quenching zone, variation of the conditional mean dilatation $\Delta$, flame-normal strain rate $s_{n}$, and flame-tangential strain rate $s_{t}$ on different $c$ isosurfaces from $c=0.05$ to $c=0.95$ for (a) the entire sample, (b) the sample beyond the influence zone, (c) the entrained elements in the quenching zone, and (d) the head-on elements in the quenching zone.

cases $6 \mathrm{~A}$ and $8 \mathrm{~A}$ to $10 \mathrm{~A}$ ) leads to the larger mean strain rate, and the tangential strain rate $s_{t}$ as well. For the nonadiabatic 8T5 and 8T0 cases, $s_{t}$ stops to increase at large $c$ values and even decreases. The main reason is that the wall heat loss reduces the flame dilatation part $\delta_{i j} s_{i j}$ in $s_{t}=\left(\delta_{i j}-n_{i} n_{j}\right) s_{i j}$.

\section{ANALYSIS OF THE COLD WALL CASE}

Within the quenching zone, where heat release from reaction starts to vanish, the flame undergoes a regime change from the typical "thin flamelet" regime to a "thickened wrinkled" regime [6] and accordingly low Damköhler number effects become increasingly prominent $[41,45]$. The cold wall case $8 \mathrm{~T} 0$ is especially interesting because of the largest wall heat loss. Figures 9(a)-9(d) compares the flame dilation and the decomposed components, i.e., the tangential strain rate $s_{t}$ and the normal strain rate $s_{n}$, for the entire flame, the flame outside the influence zone, the entrained flame part in the quenching zone and the head-on flame part in the quenching zone, respectively. Because of the dominant portion of the flame outside the influence zone, Figs. 9(a) and 9(b) are quite similar. The $\Delta$ and $s_{n}$ curves in Fig. 9(c) are similar with those in Figs. 9(a) and 9(b), but with the different peak value and the location. Figures 9(c) and 9(d) demonstrate that the head-on and entrained flame parts in the quenching zone behave quite differently. For the head-on part, $s_{t}$ and $s_{n}$ mostly exhibit monotonous variation with respect to $c$, and $\Delta$ and $s_{n}$ are totally negative, instead of partly negative for the entrained flame part. These results suggest that the comparison between the entrained flame part and the head-on flame part is essentially important to understand the FWI physics.

Choosing $c=0.1, c=0.5$, and $c=0.9$ as the representative isosurfacs in the fresh reactant region, reactive region, and product region, respectively, Fig. 10 shows the conditional alignment relation $\left|\cos \left\langle\vec{\lambda}_{i}, \vec{n}\right\rangle\right|$ in the quenching zone. It can be seen that scalar normal gradient $\vec{n}$ misaligns with the intermediate axis $\vec{\lambda}_{2}$. For the entrained part presented in Fig. 10(a), $\vec{n}$ aligns with the most compressive axis $\vec{\lambda}_{3}$ on the $c=0.1$ isosurface (the fresh gas side), while on the $c=0.5$ and $c=0.9$ isosurfaces this alignment weakens. For the head-on part shown in Fig. 10(b), $\vec{n}$ aligns well with the most compressive axis $\vec{\lambda}_{3}$ in different regions, which is consistent with the nonreactive turbulence case. Such differences indicate that flame dilatation effect is still influential even in the quenching zone. However, this influence is stronger for the entrained flame part than for the head-on flame part. The previous work by Chakraborty and Swaminathan [13] and Chakraborty et al. [16] showed that the extent of the alignment of scalar gradient with the most extensive principal strain rate increases with increasing Da because the effects of strain rate due to flame normal acceleration strengthen with increasing $\mathrm{Da}$. The flame quenching implies that the chemical time scale increases, leading to a significant drop in Da. This suggests that the flame normal vector is expected to align with the most compressive principal strain rate when the flame is quenching due to weak influences of strain rate under small values of Damköhler number. This can be substantiated from the preferential collinear 

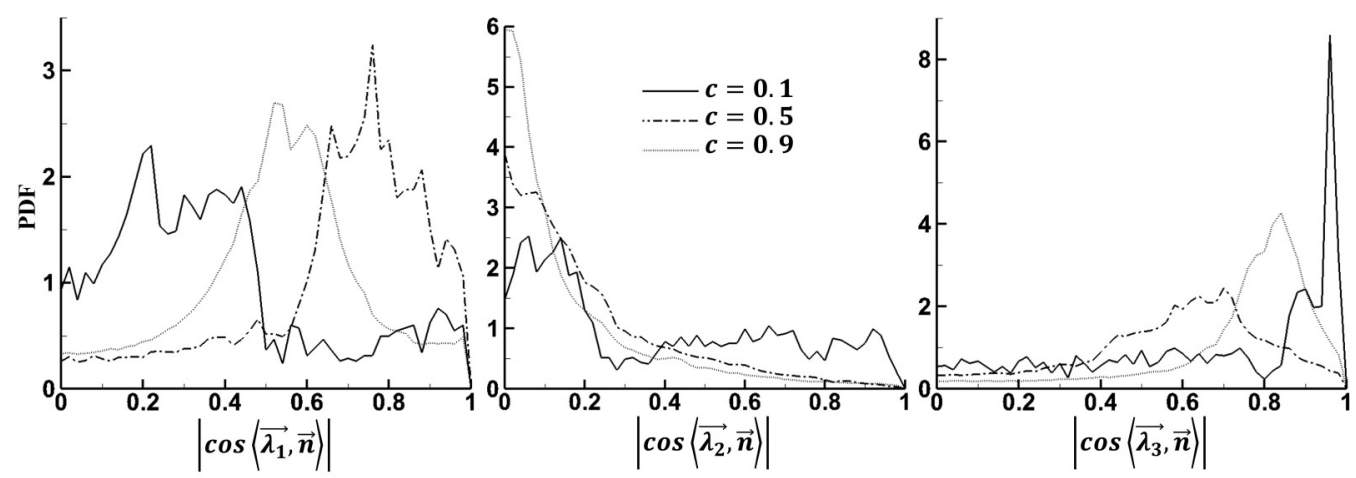

(a)
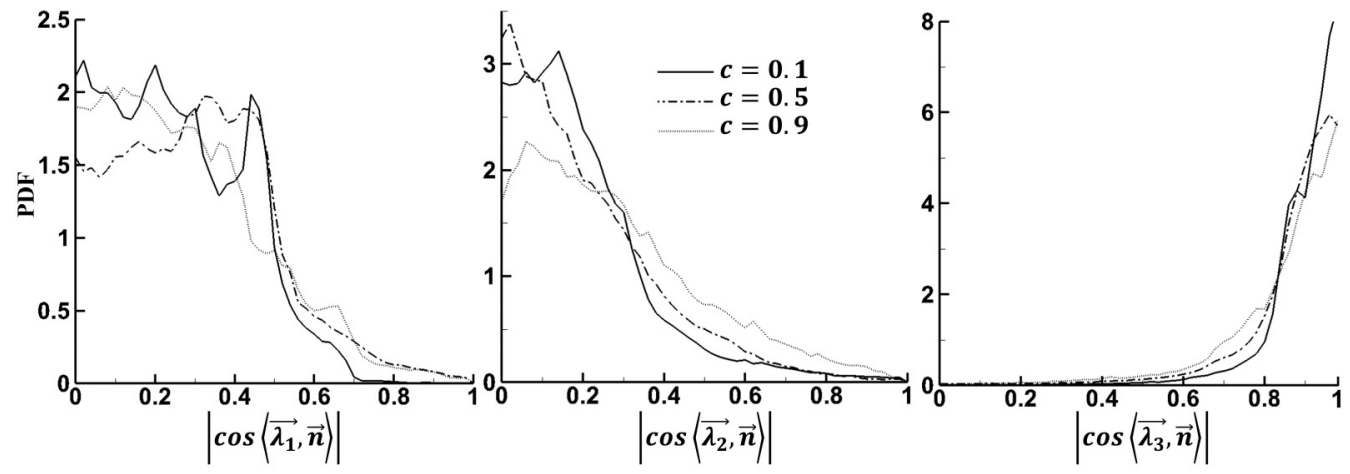

(b)

FIG. 10. PDFs of $\left|\cos \left\langle\vec{\lambda}_{i}, \vec{n}\right\rangle\right|$ on flame isosurface of $c=0.1, c=0.5$ and $c=0.9$ in case 8T0 for (a) the entrained part in the quenching zone and (b) the head-on part in the quenching zone.

alignment between $\vec{n}$ and $\vec{\lambda}_{3}$ in both entrained and head-on elements in Fig. 10. A comparison reveals that the probability of finding collinear alignment between $\vec{n}$ and $\vec{\lambda}_{3}$ is greater in the head-on part due to the stronger flame quenching effects [see the smaller magnitudes of $\Delta$ for the head-on part than in the entrained part in Figs. 9(c) and 9(d)] and thus the head-on part is expected to exhibit stronger effects of small values of Damköhler number than the entrained part. Thus predominant alignment of $\vec{n}$ and $\vec{\lambda}_{3}$ in the head-on part yields negative mean values of $s_{n}=\Lambda_{i} \cos ^{2}\left\langle\vec{n} \cdot \vec{\lambda}_{i}\right\rangle$ conditional on $c$, whereas this behavior is relatively weak in the entrained part where some positive mean values of $s_{n}$ can be discerned [see Figs. 9(c) and 9(d)].

Figure 11 shows the $\vec{n}$ and $\vec{\omega}$ alignment conditional on the $c$ isosurfaces in the quenching zone. Overall $\vec{n}$ misaligns with $\vec{\omega}$. Although vorticity $\vec{\omega}$ predominantly aligns with the intermediate principal axis $\vec{\lambda}_{2}$, it shows some extent of alignment with most extensive principal axis $\vec{\lambda}_{1}$ where dilatation effects are strong [19,32]. It can be seen from Fig. 9 that the dilatation effects are stronger in the entrained flame part than in the head-on flame part; thus $\vec{\omega}$ exhibits some degree of alignment with the most extensive principle axis $\vec{\lambda}_{1}$ and flame normal vector $\vec{n}$ (see Fig. 10). Thus, the misalignment between $\vec{\omega}$ and $\vec{n}$ is stronger in the head-on part than in the entrained part for the unburned gas side and in the reaction zone, but the tendency is reversed on the burned gas side.

The PDFs $\left|\cos \left\langle\vec{\lambda}_{i}, \vec{n}\right\rangle\right|$ shown in Fig. 12 for case $8 \mathrm{~T} 0$ are conditioned on the flame temperature on the $c=0.85$ isosurface. High-temperature zones are expected to show stronger chemical reactivity and thus the effects of high Damköhler number (i.e., strong tendency of both $\vec{n}$ and $\vec{\omega}$ to align with $\left.\lambda_{1}\right)$ are expected to be prominent in the high-temperature zones, whereas low Damköhler number 


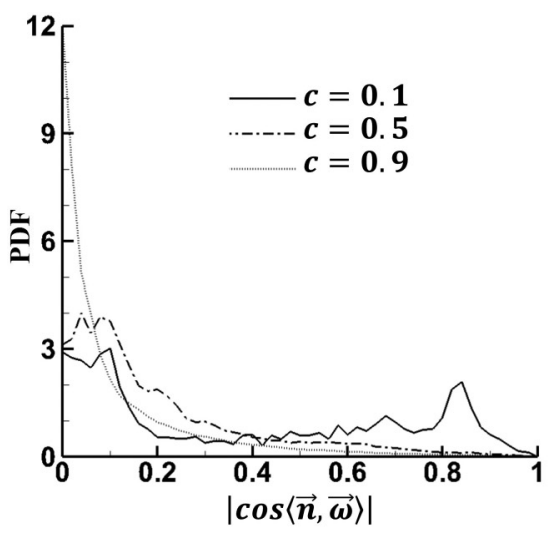

(a)

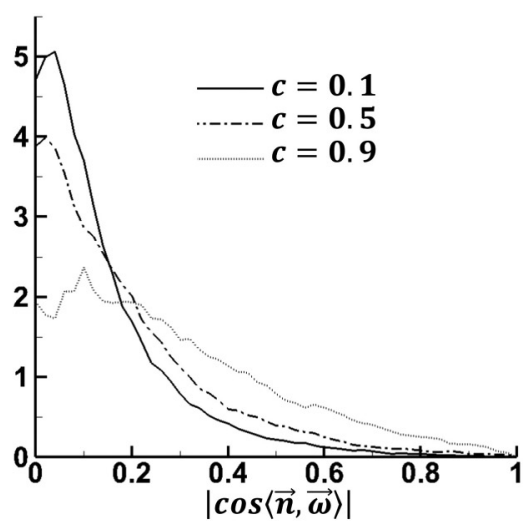

(b)

FIG. 11. PDFs of $|\cos \langle\vec{n}, \vec{\omega}\rangle|$ for (a) the entrained parts and (b) the head-on parts of case $8 \mathrm{~T} 0$ in the quenching zone of FWI.

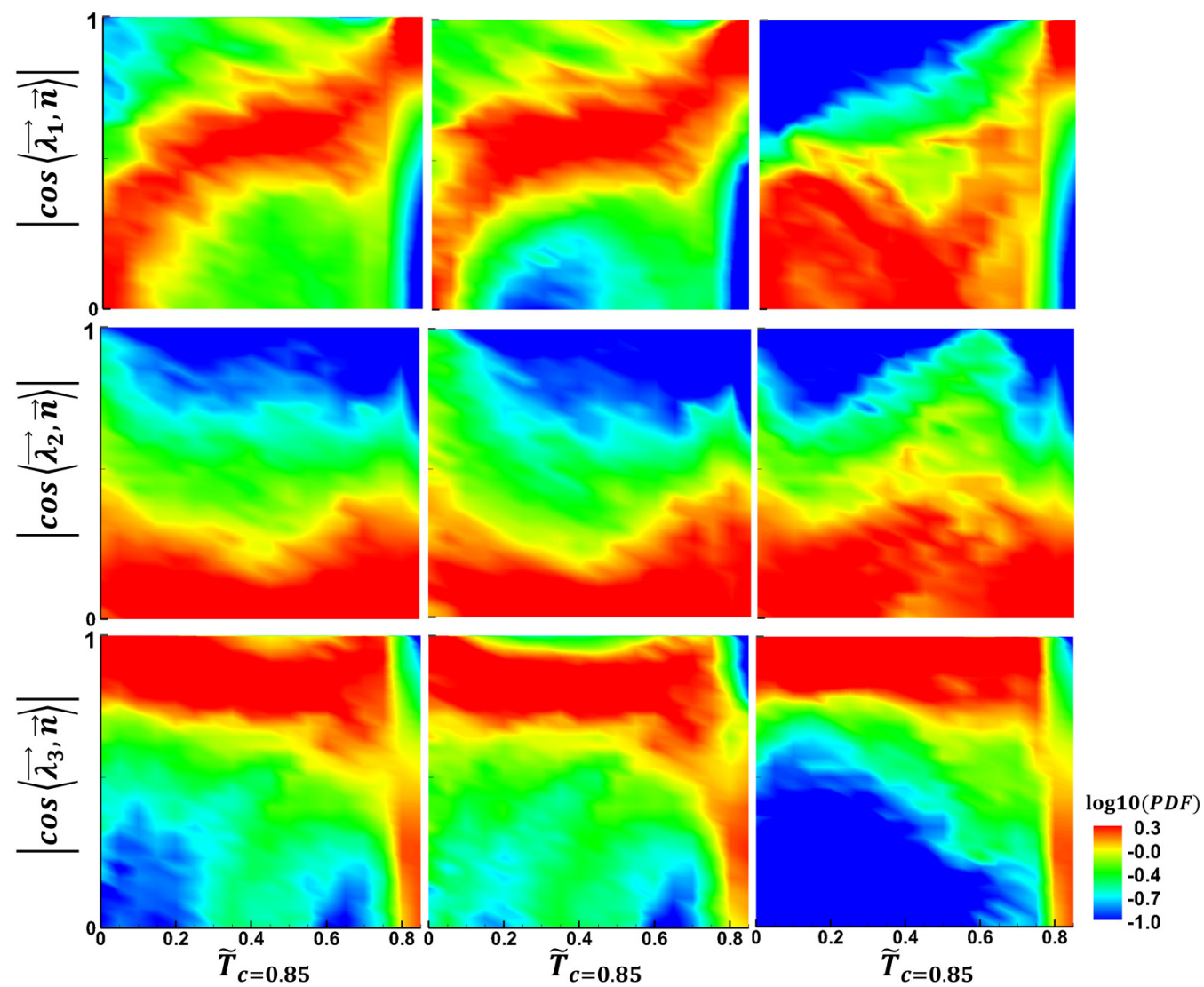

(a)

(b)

(c)

FIG. 12. PDFs of $\left|\cos \left\langle\vec{\lambda}_{i}, \vec{n}\right\rangle\right|$ conditioned on the different temperature $\tilde{T}_{c=0.85}$ of $c=0.85$ isosurfac for (a) the entire elements, (b) the entrained flame part, and (c) the head-on part of case 8T0. 
effects (i.e., strong tendency of both $\vec{n}$ and $\vec{\omega}$ to align with $\lambda_{3}$ and $\lambda_{2}$, respectively) are associated with low-temperature zones. The clear difference between Figs. 12(b) and 12(c) is that for the entrained flame part the flame normal $\vec{n}$ changes continuously to misalign with the most extensive axis $\vec{\lambda}_{1}$ once the flame temperature is lower than 0.8 , while for the head-on elements such change is more abrupt.

\section{CONCLUSIONS}

Three-dimensional DNS of turbulent flame-wall interaction (FWI) has been studied based on a statistically stationary head-on flame quenching configuration. The wall heat flux, together with the relative strengths of dilatation and turbulence perturbation, play important roles in determining the near-wall flame structure and dynamics. The present work focuses on the alignment relations among some important vector variables, including the flame normal vector, vorticity vector, the principal axes of the strain rate tensor. With the increase of wall heat loss, the flame front tends to be more broken. From the dependence of the flame temperature on the flame-wall distance, the "influence zone" and "quenching zone" in FWI can be identified. According to the relative orientation between the flame normal and the wall normal, the flame front can be categorized as the entrained part and the head-on part.

For all the cases considered here, beyond the influence zone the flame is not influenced by the wall heat loss and the flame normal aligns preferentially with the most extensive principal axis, as expected for high values of the Damköhler number. However, in the fresh gas side and the product side with negligible heat release rate, the flame normal tends to align with the most compressive principal axis, in accordance with the expectation associated with low Damköhler number combustion. For the nonadiabatic case, with the increase of the wall heat loss, the flame normal and tangential strain rates for the entrained and head-on flame parts become more different. Moreover, the misalignment of flame normal with vorticity becomes stronger as the flame approaches the adiabatic wall. Numerical results suggest that the wall heat flux does not change the misalignment relation between the flame normal and vorticity.

The cold wall case, especially in quenching zone, exhibits the strongest wall interference due to the possibility of local flame quenching. Statistics on various $c$ isosurfaces shows an overall misalignment between the flame normal and vorticity. For the head-on flame part, alignment conditioned on the flame temperature shows a sharp change of the flame normal orientation from aligning with the most extensive principal axis when $\widetilde{T}_{c=0.85} \geqslant 0.8$ to aligning with the most compressive principal axis when $\tilde{T}_{c=0.85}<0.8$. For the entrained flame elements, such change is more continuous, which indicates that in the quenching zone the entrained part is relatively less influenced by the wall condition than the head-on part. Thus, it might be necessary to delineate the physical processes occurring in the head-on and entrained parts of the quenching zone in order to propose high-fidelity modeling of scalar-turbulence interaction and vortex-stretching terms of the scalar dissipation rate and enstrophy transports, respectively, in the presence of FWI.

\section{ACKNOWLEDGMENTS}

This work is sponsored by the National Natural Science Foundation of China (under Grant No. 91441116). L.W. thanks the support of the high performance computer center ( $\pi$ ) at Shanghai Jiao Tong University. N.C. is grateful to EPSRC (EP/R029369/1, EP/P022286/1) and ARCHER for financial and computational support.

[1] N. Peters, The turbulent burning velocity for large-scale and small-scale turbulence, J. Fluid Mech. 384, 107 (1999).

[2] E. R. Hawkes and R. S. Cant, Physical and numerical realizability requirements for flame surface density approaches, Combust. Theory Model. 5, 699 (2001). 
[3] D. Veynante and L. Vervisch, Turbulent combustion modeling, Prog. Energy Combust. Sci. 28, 193 (2002).

[4] H. Pitsch, Large eddy simulation of turbulent combustion, Annu. Rev. Fluid Mech. 38, 453 (2006).

[5] T. Poinsot, D. C. Haworth, and G. Bruneaux, Direct simulation and modeling of flame-wall interaction for premixed turbulent combustion, Combust. Flame 95, 118 (1993).

[6] A. Gruber, R. Sankaran, E. R. Hawkes, and J. H. Chen, Turbulent flame-wall interaction: A direct numerical simulation study, J. Fluid Mech. 658, 5 (2010).

[7] G. Bruneaux, T. Poinsot, and J. H. Ferziger, Premixed flame-wall interaction in a turbulent channel flow: Budget for the flame surface density evolution equation and modelling, J. Fluid Mech. 349, 191 (1997).

[8] J. Lai and N. Chakraborty, Statistical behavior of scalar dissipation rate in head-on quenching of turbulent premixed flames: A direct numerical simulation analysis, Combust. Sci. Technol. 188, 250 (2016).

[9] P. Zhao, L. Wang, and N. Chakraborty, Analysis of the flame-wall interaction in premixed turbulent combustion, J. Fluid Mech. 848, 193 (2018).

[10] P. Zhao, L. Wang, and N. Chakraborty, Strain rate and flame orientation statistics in the near-wall region for turbulent flame-wall interaction. Combust. Theory Model. 22, 921 (2018).

[11] C. Dopazo, J. Martin, and J. Hierro, Local geometry of isoscalar surfaces, Phys. Rev. E 76, 056316 (2007).

[12] N. Swaminathan and R. W. Grout, Interaction of turbulence and scalar fields in premixed flames, Phys. Fluids 18, 045102 (2006).

[13] N. Chakraborty and N. Swaminathan, Influence of the Damköhler number on turbulence-scalar interaction in premixed flames. I. Physical insight, Phys. Fluids 19, 045103 (2007).

[14] N. Chakraborty and N. Swaminathan, Influence of the Damköhler number on turbulence-scalar interaction in premixed flames. II. Model development, Phys. Fluids 19, 045104 (2007).

[15] G. Hartung, J. Hult, C. F. Kaminski, J. W. Rogerson, and N. Swaminathan, Effect of heat release on turbulence and scalar-turbulence interaction in premixed combustion, Phys. Fluids 20, 035110 (2008).

[16] N. Chakraborty, M. Klein, and N. Swaminathan, Effects of lewis number on the reactive scalar gradient alignment with local strain rate in turbulent premixed flames, Proc. Combust. Inst. 32, 1409 (2009).

[17] N. Chakraborty, I. Konstantinou, and A. Lipatnikov, Effects of Lewis number on vorticity and enstrophy transport in turbulent premixed flames, Phys. Fluids 28, 015109 (2016).

[18] A. N. Lipatnikov, S. Nishiki, and T. Hasegawa, A direct numerical study of vorticity transformation in weakly turbulent premixed flames, Phys. Fluids 26, 105104 (2014).

[19] N. Chakraborty, Statistics of vorticity alignment with local strain rates in turbulent premixed flames, Eur. J. Mech. B 46, 201 (2014).

[20] J. Lai, N. Chakraborty, and A. Lipatnikov, Statistical behaviour of vorticity and enstrophy transport in head-on quenching of turbulent premixed flames, Eur. J. Mech. B 65, 384 (2017).

[21] W. T. Ashurst, A. R. Kerstein, R. M. Kerr, and C. H. Gibson, Alignment of vorticity and scalar gradient with strain rate in simulated Navier-Stokes turbulence, Phys. Fluids 30, 2343 (1987).

[22] P. J Diamessis and K. K. Nomura, Interaction of vorticity, rate-of-strain, and scalar gradient in stratified homogeneous sheared turbulence, Phys. Fluids 12, 1166 (2000).

[23] G. Gulitski, M. Kholmyansky, W. Kinzelbach, B. Lüthi, A. Tsinober, and S. Yorish, Velocity and temperature derivatives in high-Reynolds-number turbulent flows in the atmospheric surface layer. Part 3. Temperature and joint statistics of temperature and velocity derivatives, J. Fluid Mech. 589, 103 (2007).

[24] D. A. Donzis and P. K. Yeung, Resolution effects and scaling in numerical simulations of passive scalar mixing in turbulence, Physica D 239, 1278 (2010).

[25] K. K. Nomura and S. E. Elghobashi, The structure of inhomogeneous turbulence in variable density nonpremixed flames, Theor. Comput. Fluid Dyn. 5, 153 (1993).

[26] O. N. Boratav, S. E. Elghobashi, and R. Zhong, On the alignment of strain, vorticity and scalar gradient in turbulent, buoyant, nonpremixed flames, Phys. Fluids 10, 2260 (1998).

[27] F. A. Jaberi, D. Livescu, and C. K. Madnia, Characteristics of chemically reacting compressible homogeneous turbulence, Phys. Fluids 12, 1189 (2000).

[28] S. H. Kim and H. Pitsch, Scalar gradient and small-scale structure in turbulent premixed combustion, Phys. Fluids 19, 115104 (2007). 
[29] R. Sankaran, E. R. Hawkes, J. H. Chen, T. Lu, and C. K. Law, Structure of a spatially developing turbulent lean methane-air bunsen flame, Proc. Combust. Inst. 31, 1291 (2007).

[30] N. Chakraborty and R. S. Cant, Effects of lewis number on scalar transport in turbulent premixed flames, Phys. Fluids 21, 035110 (2009).

[31] N. Chakraborty, J. W. Rogerson, and N. Swaminathan, The scalar gradient alignment statistics of flame kernels and its modelling implications for turbulent premixed combustion, Flow Turbul. Combust. 85, 25 (2010).

[32] P. E. Hamlington, A. Y. Poludnenko, and E. S. Oran, Interactions between turbulence and flames in premixed reacting flows, Phys. Fluids 23, 125111 (2011).

[33] B. Coriton and J. H. Frank, Impact of heat release on strain rate field in turbulent premixed bunsen flames, Proc. Combust. Inst. 36, 1885 (2017).

[34] A. Tsinober, L. Shtilman, and H. Vaisburd, A study of properties of vortex stretching and enstrophy generation in numerical and laboratory turbulence, Fluid Dyn. Res. 21, 477 (1997).

[35] Z. She and E. Jackson, Intermittent vortex structures in homogeneous isotropic turbulence, Nature (London) 344, 226 (1990).

[36] A. J. Majda, Vorticity, turbulence, and acoustics in fluid flow, SIAM Rev. 33, 349 (1991).

[37] J. Jimenez, Kinematic alignment effects in turbulent flows, Phys. Fluids A 4, 652 (1992).

[38] B. W. Zeff, D. D Lanterman, R. Mcallister, R. Roy, E. J. Kostelich, and D. R. Lathrop, Measuring intense rotation and dissipation in turbulent flows, Nature (London) 421, 146 (2003).

[39] B. Luthi and A. Tsinober, Lagrangian measurement of vorticity dynamics in turbulent flow, J. Fluid Mech. 528, 87 (2005).

[40] H. Xu, A. Pumir, and E. Bodenschatz, The pirouette effect in turbulent flows, Nat. Phys. 7, 709 (2011).

[41] J. Lai and N. Chakraborty, A-priori direct numerical simulation modelling of scalar dissipation rate transport in head-on quenching of turbulent premixed flames, Combust. Sci. Technol. 188, 1440 (2016).

[42] O. A. Ezekoye and R. Greif, A comparison of one and two dimensional flame quenching: Heat transfer results, in Proceedings of the 29th National Conference and Exposition on Heat Transfer, Atlanta, GA, 8-11 August 1993 (ASME, 1993).

[43] G. Bruneaux, K. Akselvoll, T. Poinsot, and J. H. Ferziger, Flame-wall interaction simulation in a turbulent channel flow, Combust. Flame 107, 27 (1996).

[44] V. N. Kurdyumov, E. Fernandez, and A. Linan, Flame flashback and propagation of premixed flames near a wall, Proc. Combust. Inst. 28, 1883 (2000).

[45] J. Lai and N. Chakraborty, Effects of lewis number on head on quenching of turbulent premixed flames: A direct numerical simulation analysis, Flow Turb. Combust. 96, 279 (2016).

[46] S. R. Vosen, R. Greif, and C. K. Westbrook, Unsteady heat transfer during laminar flame quenching, Symp. (Intl) Combust. 20, 75 (1985).

[47] L. Connelly, T. Ogasawara, D. Lee, R. Greif, and R. F. Sawyer, Fall meeting, the Combustion Institute/Western States Section, Stanford, CA, Paper N. WSCI 93-077 (1993).

[48] P. Popp and M. Baum, Analysis of wall heat fluxes, reaction mechanisms, and unburnt hydrocarbons during the head-on quenching of a laminar methane flame, Combust. Flame 108, 327 (1997).

[49] J. Lai, M. Klein, and N. Chakraborty, Direct numerical simulation of head-on quenching of statistically planar turbulent premixed methane-air flames using a detailed chemical mechanism, Flow Turb. Combust. 101, 1073 (2018).

[50] T. Poinsot and D. Veynante, Theoretical and Numerical Combustion, 1st ed. (R. T. Edwards, New Jersey, USA, 2005).

[51] R. S. Rogallo, Numerical experiments in homogeneous turbulence, NASA STI/RECON Technical Report N 81 (1981).

[52] K. W. Jenkins and R. S. Cant, Direct numerical simulation of turbulent flame kernels, in Recent Advances in DNS and LES, edited by D. Knight and L. Sakell (Springer, Amsterdam, 1999), pp. 191-202.

[53] A. A. Wray, Minimal storage time-advancement schemes for spectral methods, Report No. M.S. 202 A-1, 1990 unpublished report, NASA Ames Research Center, California (1991). 
[54] C. S. Yoo, Y. Wang, A. Trouvé, and H. G. Im, Characteristic boundary conditions for direct simulations of turbulent counterflow flames, Combust. Theory Model. 9, 617 (2005).

[55] C. S. Yoo and H. G. Im, Characteristic boundary conditions for simulations of compressible reacting flows with multi-dimensional, viscous and reaction effects, Combust. Theory Model. 11, 259 (2007).

[56] G. Lodato, P. Domingo, and L. Vervisch, Three-dimensional boundary conditions for direct and largeeddy simulation of compressible viscous flows, J. Comput. Phys. 227, 5105 (2008). 